\title{
Circular RNA hsa-circ-000881 suppresses the progression of lung adenocarcinoma in vitro via a miR-665/PRICKLE2 axis
}

\author{
Chu Huang^, Weiming Yue, Lin Li, Shuhai Li, Cun Gao, Libo Si, Lei Qi, Chuanle Cheng, Ming Lu, \\ Guanqing Chen, Jingjing Cui, Renchang Zhao, Yongmeng Li, Hui Tian \\ Department of Thoracic Surgery, Qilu Hospital, Cheeloo College of Medicine, Shandong University, Jinan, China \\ Contributions: (I) Conception and design: C Huang; (II) Administrative support: H Tian; (III) Provision of study materials or patients: W Yue, L \\ Li, S Li, C Gao, L Si, L Qi, C Cheng, M Lu, G Chen, J Cui, R Zhao, Y Li; (IV) Collection and assembly of data: C Huang; (V) Data analysis and \\ interpretation: C Huang; (VI) Manuscript writing: All authors; (VII) Final approval of manuscript: All authors. \\ Correspondence to: Hui Tian. No.107, Wenhua West Road, Lixia District, Jinan, China. Email: tianhuiq1@126.com.
}

\begin{abstract}
Background: Circular RNA (circRNA) has become a new focus in the field of tumor biology research in recent years. Many circRNAs have been showed to play an important role in the progression of lung adenocarcinoma (LUAD). In this work, we studied the oncological role of hsa-circ-000881 in LUAD and attempted to explore the related mechanism.

Methods: The relative expressions of hsa-circ-000881, miR-665, and PRICKLE2 were detected by RTqPCR or western blot. Functional assays were conducted to analyze the role of hsa-circ-000881 in the proliferation, migration, and invasion of LUAD cells. A luciferase reporter assay was performed to verify whether hsa-circ-000881, miR-665, and PRICKLE2 interact with each other.

Results: Circ-000881 was remarkably downregulated in LUAD. Overexpression of circ-000881 attenuated cell growth, migration, and invasion, whereas its knockdown enhanced the malignancy of LUAD cells. The results of luciferase reporter assay and bioinformatics analysis confirmed that circ-000881 served as a sponge for miR-665, and PRICKLE2 was a direct target of miR-665.Overexpression of miR-665 or silencing of PRICKLE2 abolished circ-000881-mediated inhibition of malignant tumor behavior in LUAD cells.

Conclusions: Circ-000881 has inhibitory effects on LUAD via a miR-665/PRICKLE2 axis, suggesting that circ-000881 may be an underlying therapeutic target for LUAD.
\end{abstract}

Keywords: Circ-000881; miR-665; lung adenocarcinoma (LUAD); PRICKLE2

Submitted Jan 21, 2021. Accepted for publication Mar 12, 2021.

doi: $10.21037 / \mathrm{atm}-21-844$

View this article at: http://dx.doi.org/10.21037/atm-21-844

\section{Introduction}

Lung cancer is most prevalent cancer type in the world both in incidence and mortality (1). Non-small cell lung cancer (NSCLC) accounts for more than half of all lung cancer cases, with its most common subtype, lung adenocarcinoma (LUAD), comprising $40 \%$ of all lung cancers $(2,3)$. Patients with adenocarcinoma often develop distant metastases early on in their disease (4). Early diagnosis of LUAD is an issue that urgently needs to be addressed. Many patients with
LUAD missed the best time for surgical treatment when they were diagnosed (5). In the last decade, progress has been made in the treatment of LUAD, but mortality is still high (6). Biological research on molecular markers of tumor genesis and development can provide new ideas on more efficient means of diagnosis and therapeutic strategies for patients with LUAD.

Circular RNAs (circRNAs) belong to the functional noncoding RNA (ncRNA) family, the members of which have been widely studied in recent decades. With their high

\footnotetext{
^ ORCID: 0000-0003-3564-1812.
} 
level of stability and highly conserved structure, circRNAs have a low likelihood of degradation (7). Since the discovery of circRNAs, many researchers have attempted to investigate the whether or not they are involved in the occurrence and development of human diseases, especially cancers, and some studies have produced exciting results (8). Wang et al. reported that hsa-circ-0001038 was upregulated in cervical cancer, which was correlated with an unfavorable outcome for patients (9). In their study, Sang et al. observed that high expression of circRNA ciRS-7 facilitated the malignant biological behavior of esophageal squamous carcinoma cells by obviously promoting cell growth, migration, and invasion (10). In another study, down-regulation of hsa-circ-0091570 was often observed in patients with hepatocellular carcinoma who had a higher tumor grade, and accelerated tumor growth in vivo (11). Dysregulation of circRNAs, such as hsa-circ-0001588, hsa-circ-0000211, and circASPH, has also been confirmed in $\operatorname{LUAD}(2,4,12)$.

Recently, some circRNAs have been proven to possess protein translation ability (13). In cancers, circRNAs usually interact with microRNAs (miRNAs) to produce a crucial promotion or suppression effect (14). For instance, it was reported that circ-RanGAP1 sponges miR-877$3 \mathrm{p}$ to promote malignant biological behaviors in gastric cancer cells (15). The interaction between circ-MYLK and miR-29a was demonstrated by Zhong et al. to facilitate the progression of bladder carcinoma by inducing epithelialmesenchymal transition (16). In another study, circ-0000977 was also found to regulate the progression of pancreatic ductal adenocarcinoma through interaction with miR874-3p (17). However, so far, the role of circRNA in the progression of LUAD has remained unclear.

In the present research, we analyzed the biological function of circRNA circ-000881 in LUAD, along with the underlying mechanism, using The Cancer Genome Atlas (TCGA) database.

We present the following article in accordance with the MDAR reporting checklist (available at http://dx.doi. org/10.21037/atm-21-844).

\section{Methods}

\section{Patients and tissue samples}

A total of 40 pairs of LUAD and para-carcinoma normal tissues were collected from patients who had undergone surgery at our hospital. None of the patients had a history of other tumors or had received radiotherapy or chemotherapy preoperatively. All tissue samples were immediately frozen in liquid nitrogen and stored in a freezer at $-80{ }^{\circ} \mathrm{C}$. Ethics approval for this study was acquired from the Medical Ethics Committee of Qilu hospital, and informed consent was obtained from all participants. All procedures performed in this study involving human participants were in accordance with the Declaration of Helsinki (as revised in 2013).

\section{Bioinformatic analysis}

We used the Cancer Genome Atlas (TCGA, https://portal. gdc.cancer.gov/) database to analyze circ-000881 and miR665 expression in tumor and normal tissues. We used TargetScan (http://www.targetscan.org/) to predict possible binding sites between circ-000881 and miR-665. Possibly target genes of miR-665 were predicted by using Starbase (http://starbase.sysu.edu.cn/), The Cancer Genome Atlas (TCGA), TargetScan and miRDB (http://mirdb.org/).

\section{Cell culture}

Human bronchial epithelial cells (HBE) and lung cancer cells (A549, HCC827, H23, H1975, and H1299) were obtained from the Institute of Biochemistry and Cell Biology of the Chinese Academy of Science (Shanghai, China). All cells were cultured in Dulbecco's Modified Eagle Medium (DMEM; KeyGEN, Jiangsu, China) containing $10 \%$ fetal bovine serum (FBS, GIBCO, Shanghai, China) in a incubator at $37^{\circ} \mathrm{C}$ with $5 \% \mathrm{CO}_{2}$.

\section{Reverse transcription and quantitative polymerase chain reaction ( $R T-q P C R)$}

Total RNA was extracted from tissues or cultured cells with TRIzol reagent (Invitrogen, CA, USA). Subsequently, reverse transcription was performed to obtain cDNA by using the PrimeScript 1st strand cDNA Synthesis Kit (TaKaRa, Dalian, China). Then, Quantitative realtime PCR (qRT-PCR) was accomplished using the SYBR Premix Ex Taq II kit (Takara). U6 (for miR-665) and GAPDH (for circ-00081 and PRICKLE2) were used as an internal control. The $2^{-\Delta \Delta C t}$ method was chosen to calculate the relative messenger RNA (mRNA) expression. The sequences of primers are listed in Table 1 .

\section{Western blot}

Cellular protein was extracted with radioimmunoprecipitation assay (RIPA) buffer (Beyotime, Shanghai, China). A BCA 
protein assay kit (Tiangen, Beijing, China) was employed to determine protein concentration. Equal amounts of protein were denatured, separated by $10 \%$ sodium dodecyl sulfate-polyacrylamide gel electrophoresis (SDS-PAGE), and transferred to a polyvinylidene difluoride (PVDF) membrane (Millipore, Bedford, MA, USA). After blocking with $5 \%$ nonfat skim milk at room temperature for 1 hour, the membranes were incubated with primary antibody overnight at $4{ }^{\circ} \mathrm{C}$. Next, the membrane was incubated with the secondary antibody at room temperature for 1 hour. The protein bands were visualized by enhanced chemiluminescence (ECL, Thermo Fisher Scientific). The quantities of the protein bands were analyzed by ImageJ software. The used antibodies are listed in Table 2.

\section{Cell transfection}

Circ-000881 siRNA, PRICKLE2 siRNA，miR-665 mimics, and their negative controls were purchased from GenePharma (Shanghai, China). To overexpress circ-000881,

Table 1 The sequences of primers were used in this study

\begin{tabular}{lc}
\hline Name & \multicolumn{1}{c}{ Sequences } \\
\hline miR-665 & Forward: 5'-TTAGAGGCAGAGACCCCCCGT-3' \\
& Reverse: 5'-TGGGTAGAGGCATAGACCCGT-3' \\
U6 & Forward: 5'-CCCTTCGGGGACATCCGATA-3' \\
& Reverse: 5'-TTTGTGCGTGTCATCCTTGC-3' \\
circ-00081 & Forward: 5'-AGGGACCGAACGGACTGTAG-3' \\
& Reverse: 5'-ACAAAACCGTAACAGCGAGC-3' \\
PRICKLE2 & Forward: 5'-CTGCACAAAGGCCCTCTCAG-3' \\
& Reverse: 5'-CTGCGGGCTAACAGAGAAGT-3' \\
GAPDH & Forward: 5'-GCAACTAGGATGGTGTGGCT-3' \\
& Reverse: 5'-TCCCATTCCCCAGCTCTCATA-3' \\
\hline
\end{tabular}

the sequence of circ-000881 was introduced into pcDNA3.1 vector (Geneseed, Guangdong, China). Transfection was performed using Lipofectamine 3000 (Invitrogen). The transfection efficiency was evaluated by RT-qPCR, and the cells were collected for subsequent experiments.

\section{Cell proliferation assays}

After transfection, a cell counting Kit-8 (CCK-8) assay was performed. First, A549 and HCC827 cells were seeded into 96-well plates and incubated at $37{ }^{\circ} \mathrm{C}$ with $5 \% \mathrm{CO}_{2}$ for the indicated times ( $0,24,48$, and 72 hours). Then, the cells were treated with CCK-8 reagent (Dojindo, Mashikimachi, Japan) for 2 hours. The optical density value was detected using a microplate reader at $450 \mathrm{nM}$. A colony formation assay was also performed to assess cell proliferation. Cells (500 cells/well) were seeded into 6-well plates and cultured in an incubator $\left(37^{\circ} \mathrm{C}, 5 \% \mathrm{CO}_{2}\right)$. After 14 days of incubation, $4 \%$ paraformaldehyde was used to fix the cells, which were then stained with $1 \%$ crystal violet. Finally, a microscope was used to count the number of cell clones.

\section{Wound-bealing assay}

A wound-healing experiment was used to detect the migration ability of cells. Briefly, after transfection, HCC827 and A549 cells were seeded in 6-well plates and then incubated with serum-free DMEM. When cell confluency reached $90 \%$, the monolayer was scratched with a sterile pipette tip. The wounds were observed and photographed at 0 and 48 hours, and Image J software was used to analyze the migration rate.

\section{Transwell assay}

After transfection, A549 and HCC827 cells $\left(1 \times 10^{5}\right.$ cells $)$ were seeded into the upper chambers, which had been

Table 2 The antibodies were used in this study

\begin{tabular}{ll}
\hline Antibody & \multicolumn{1}{c}{ Supplier name } \\
\hline Primary antibody & \\
PRICKLE2 & ab211419; Abcam, Cambridge, UK \\
GAPDH & ab8245, Abcam \\
Secondary antibody & \\
HRP-labeled Goat Anti-Rabbit IgG(H+L) & A0208; Beyotime, China \\
HRP-labeled Goat Anti-Mouse $\operatorname{lgG}(\mathrm{H}+\mathrm{L})$ & A0216; Beyotime, China \\
\hline
\end{tabular}


coated with Matrigel (BD Biosciences, New York, NY, USA) and filled with serum-free medium. Medium supplemented with $10 \%$ FBS was added to the lower compartment. After incubation for 2 days, the cells on the lower surface were fixed and stained with $0.1 \%$ crystal violet for $15 \mathrm{~min}$. Nonmigrated cells were wiped away, and migrated cells were counted under a microscope in high power fields.

\section{Luciferase reporter assay}

A549 and HCC827 cells $\left(1 \times 10^{4}\right.$ cells per well $)$ were seeded into 96-well plates. Circ-000881 or PRICKLE2 wildtype and mutant luciferase reporter plasmids (QIAGEN, Shanghai, China) were constructed. Each kind of plasmids was subsequently co-transfected with miR-665 mimic or mimic NC into A549 and HCC827 cells. After 48 hours of transfection, a dual-luciferase reporter assay (Promega) was carried out to assess luciferase activity.

\section{Statistical analysis}

We conducted statistical analyses using the GraphPad Prism 7 (GraphPad Software Inc., San Diego, CA, USA) software, and data were expressed as means \pm standard deviations. Statistical differences were analyzed using Student's $t$-test, one-way analysis of variance (ANOVA), or Pearson's correlation. $\mathrm{P}<0.05$ was regarded as a statistically significant difference.

\section{Results}

\section{Circ-000881 was downregulated in LUAD tissues and cells}

First, TCGA database analysis showed circ-000881 expression to be downregulated in LUAD tissues $(\mathrm{P}=0.0012$, Figure $1 A$ ). Therefore, we collected 40 pairs of LUAD tissues and adjacent normal tissues to measure circ-000881 expression with RT-qPCR. The results indicated that circ-000881 was expressed at a low level in tumor tissues compared with para-carcinoma tissues $(\mathrm{P}<0.01$, Figure $1 B)$. The expression of circ-000881 was also assessed in HBE and A549, HCC827, H23, H1975, and H1299 cells. Compared with that in HBE cells, circ-000881 expression was downregulated in A549, HCC827, H23, H1975, and H1299 cells $(\mathrm{P}<0.01$, Figure $1 C)$.

\section{Circ-000881 acted as a suppressor in A549 and HCC827 cells}

Among the lung cancer cells, HCC827 cells and A549 cells exhibited low and high levels of circ-000881 expression, respectively. Therefore, we selected A549 cells for circ-000881 silencing and HCC827 for circ-000881 overexpression. RT-qPCR confirmed that the transfection was successful (Figure 2A). Next, we examined the effects of circ-000881 on the biological functions of HCC827 and A549 cells. The CCK-8 assay results demonstrated that after the transfection of overexpression vector, the proliferative capabilities of HCC827 cells were remarkably decreased, while after transfection with si-circ-000881, A549 cells became obviously less proliferative $(\mathrm{P}<0.01$, Figure $2 B$ ). The outcomes of colony formation assays arrived at the same conclusion $(\mathrm{P}<0.01$, Figure $2 C)$. Furthermore, overexpression of circ-000881 diminished the migratory abilities and invasive capabilities of HCC 827 cells, while circ-000881-silenced A549 cells became more invasive and could migrate more easily $(\mathrm{P}<0.01$, Figure $2 D, E)$.

\section{Circ-000881 interacted with miR-665 in LUAD}

To further explore how circ-000881 achieves its inhibitory effect in LUAD, we investigated whether circ-000881 contains the binding sequence of miR-665 using a bioinformatics tool (TargetScan 7.2). The result confirmed that circ-000881 contains the binding sites of miR665 (Figure 3A). The luciferase reporter assay results demonstrated that miR-665 mimic reduced the luciferase activity of A549 and HCC817 cells transfected with circ000881 wild-type luciferase reporter plasmids $(\mathrm{P}<0.01$, Figure $3 B$ ), but the same effect was not observed in the mutant group. Moreover, analysis of the TGCA database showed miR-665 expression to be increased in many cancers, including LUAD, which suggested that it might play a role in tumor promotion (Figure 3C). Subsequently, we detected miR-665 expression at the RNA level both in clinical LUAD samples and in para-carcinoma tissues. MiR665 was highly expressed in LUAD tissues but expressed at low levels in para-carcinoma tissues $(\mathrm{P}<0.01$, Figure $3 D)$. A high expression of miR-665 was accompanied by a low expression of circ-000881 in LUAD tissues, suggesting there to be a negative regulatory mechanism between them $(\mathrm{P}=0.0004$, Figure $3 E)$. Together, these results suggested that circ-000881 served as a sponge for miR-665 in LUAD.

\section{PRICKLE2 is a direct target of miR-665}

By using bioinformatics tools (Starbase, TCGA, TargetScan, and miRDB), we found that MOCS1, 


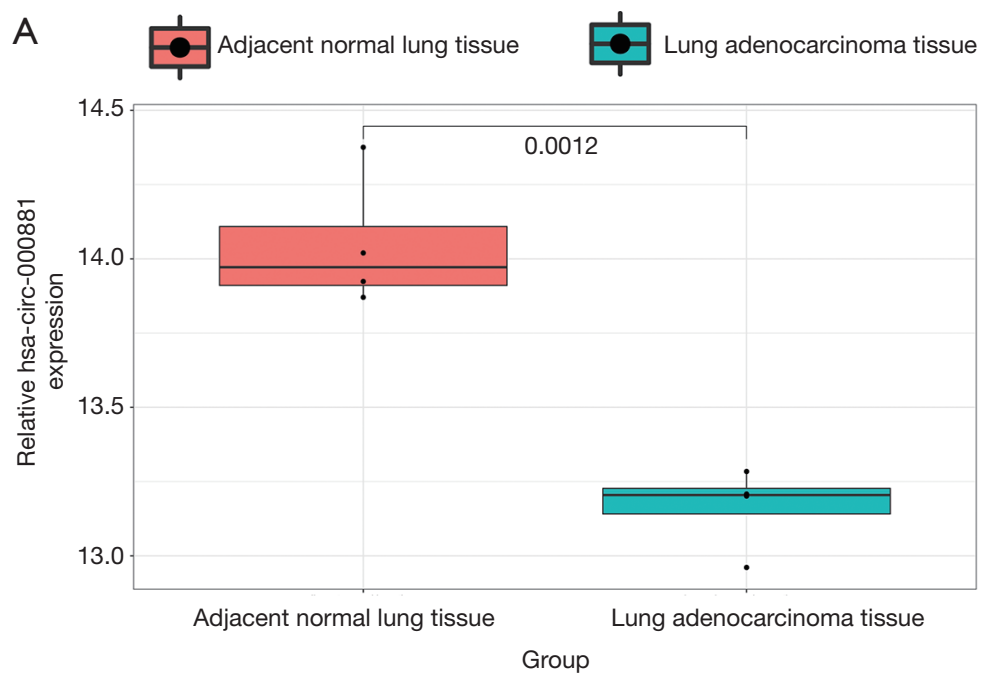

\section{B}

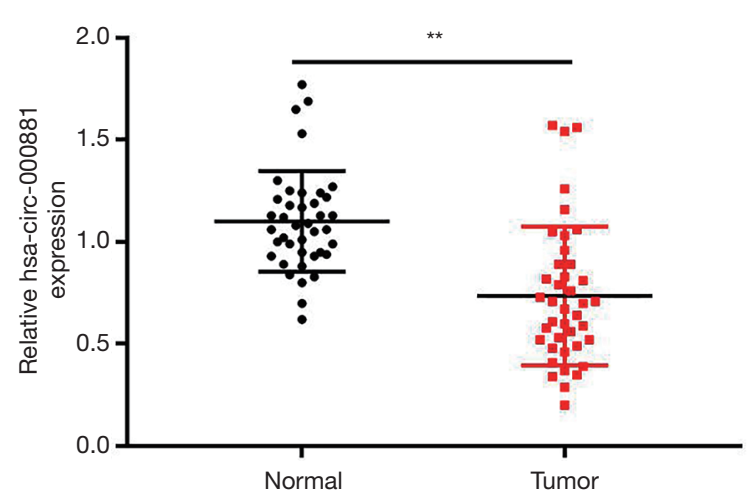

C

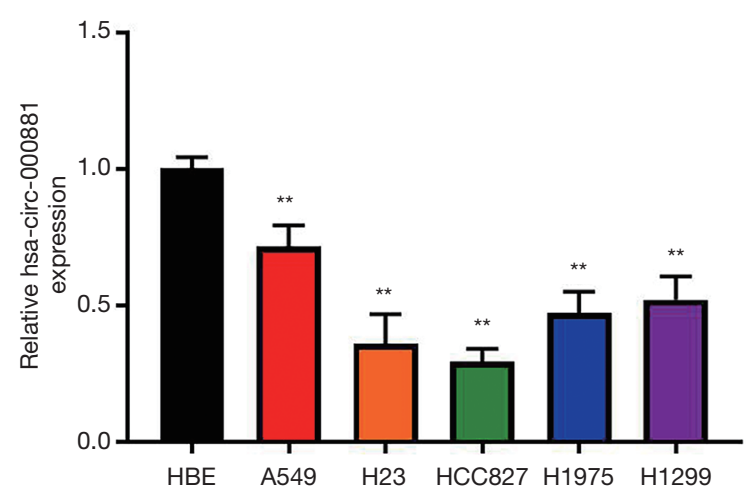

Figure 1 Circ-000881 is downregulated in lung adenocarcinoma (LUAD) tissues and cells. (A) The Cancer Genome Atlas (TCGA) analysis showed tumor tissues to have lower circ-000881 expression than normal tissues. (B) RT-qPCR was used to measure circ-000881 expression in 40 pairs of LUAD tissues and para-carcinoma tissues. (C) RT-qPCR was used to measure circ-000881 expression in human bronchial epithelial cells (HBE) and non-small cell lung cancer (NSCLC) cells (HCC827, H23, A549, H1975, and H1299). **P<0.01 vs. Normal or HBE group.

CDC25A, PRELP, PRICKLE2, KCNN3, and TGFBR2 might be potential targets of miR-665 (Figure 4A). Among these genes, PRICKLE2 has rarely been the focus of studies on cancer, which intrigued us enormously. Therefore, we immediately investigated the potential interaction between miR-665 and PRICKLE2. Figure $4 B$ shows the potential binding sites of miR-665 and PRICKLE2. The luciferase reporter assay showed that miR-665 mimic reduced the luciferase activity of the PRICKLE2 wild-type luciferase reporter plasmids in A549 and HCC817 cells $(\mathrm{P}<0.01$, Figure $4 C$ ), but had no significant effect on the mutated luciferase reporter plasmids, indicating that miR-665 could bind to PRICKLE2. This observation indicated that miR-
665 could bind to PRICKLE2. Furthermore, analysis of the expression of PRICKLE2 in LUAD was carried out using data from the TCGA database. PRICKLE2 had a low expression in LUAD tissues, and patients with higher expression of PRICKLE2 displayed longer overall survival (Figures 4D,E), which suggested that PRICKLE2 might play a tumor-inhibitor role. PRICKLE2 expression was significantly decreased in LUAD tissues $(\mathrm{P}<0.01$, Figure $4 F)$. Furthermore, the expression of PRICKLE2 was negatively correlated with miR-665 in LUAD tissues $(\mathrm{P}<0.0001$, Figure $4 G$ ). From these observations, it is reasonable to draw the conclusion that miR-665 can bind to PRICKLE2 directly in LUAD. 
A

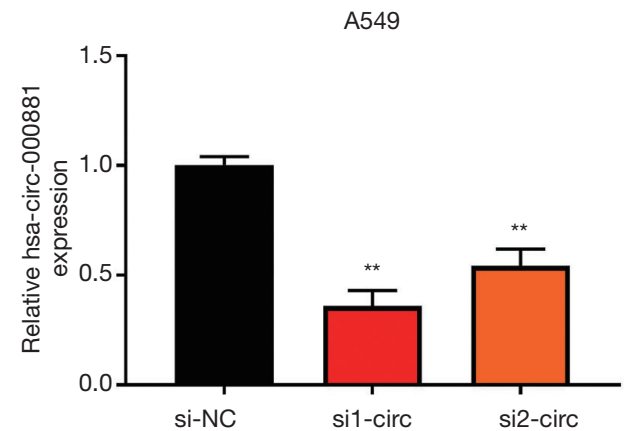

B

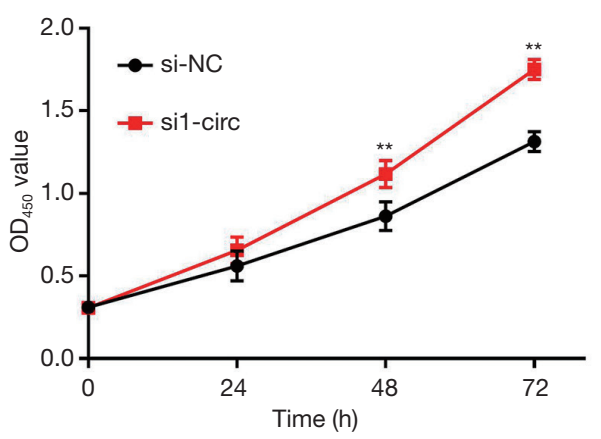

C

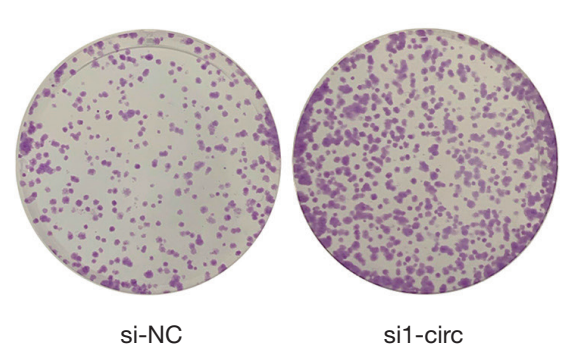

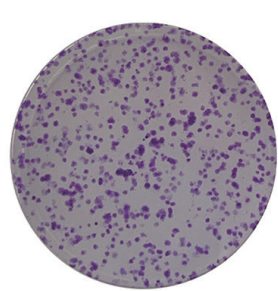

Vector
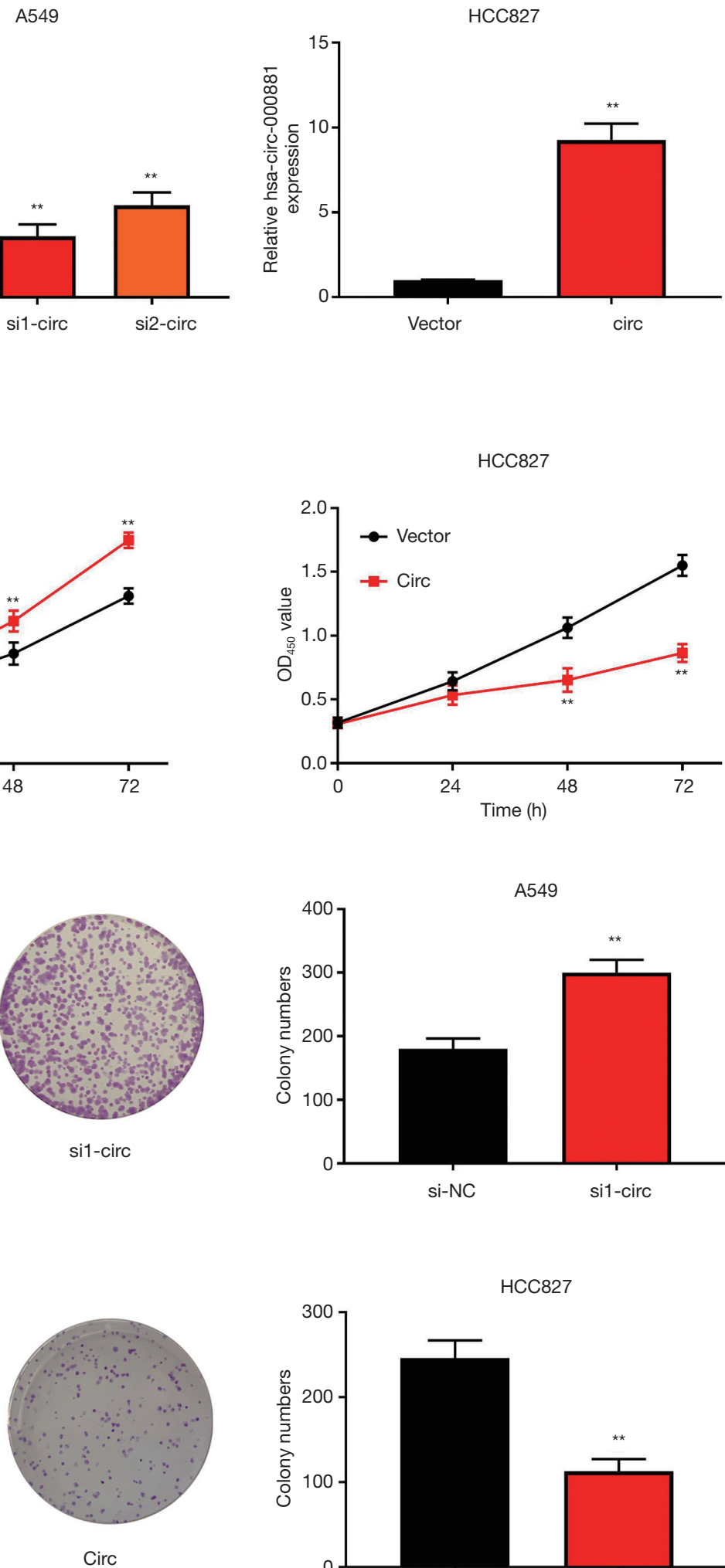

HCC827
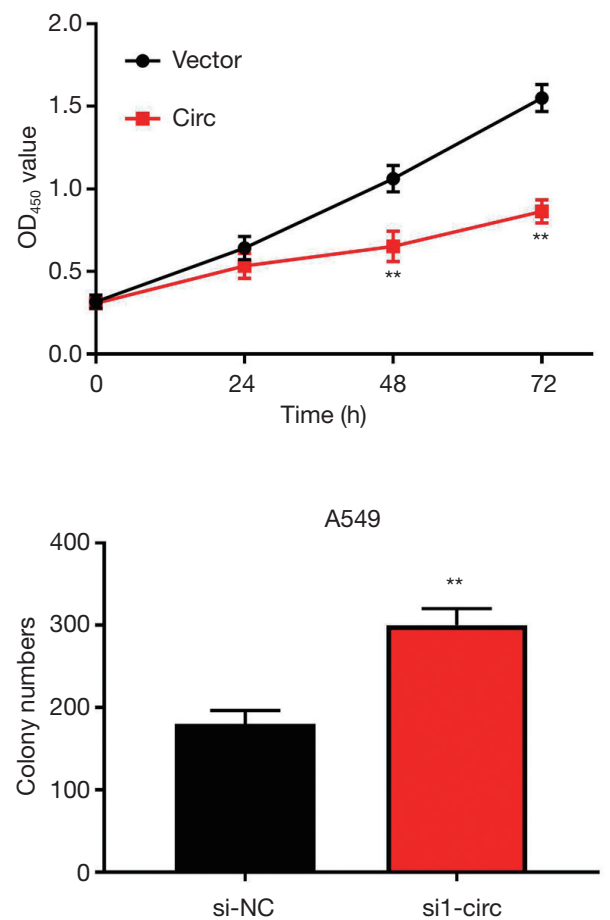

HCC827

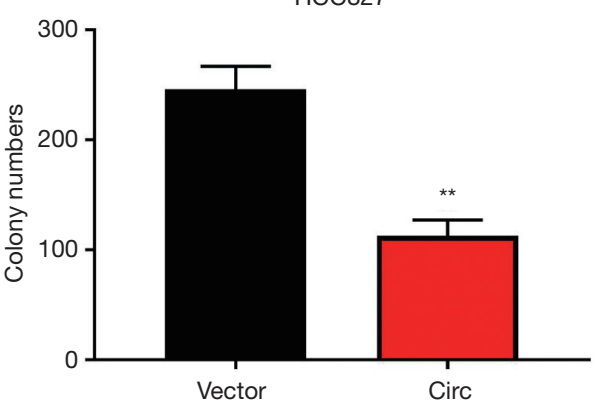


D
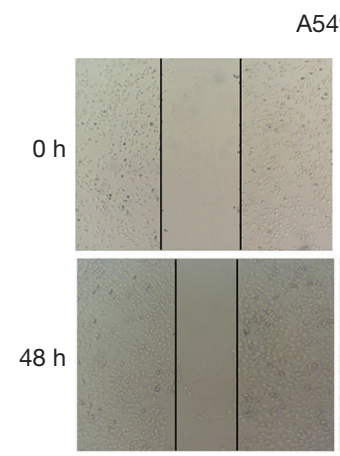

si-NC

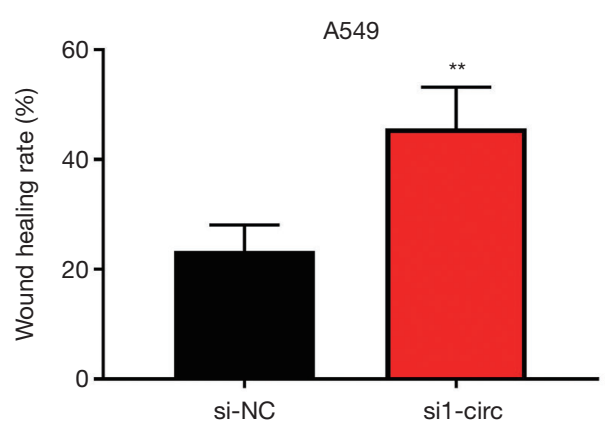

$\mathrm{E}$

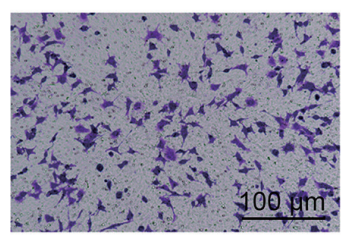

si-NC
A549
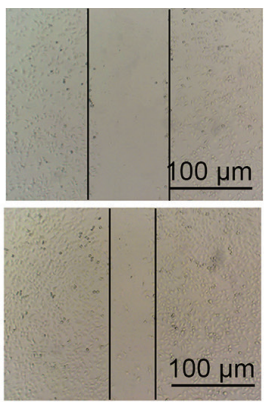

si1-circ

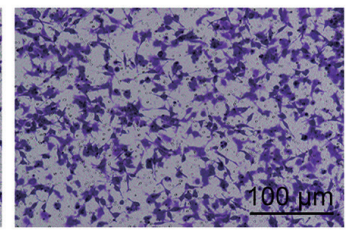

si1-circ
HCC827

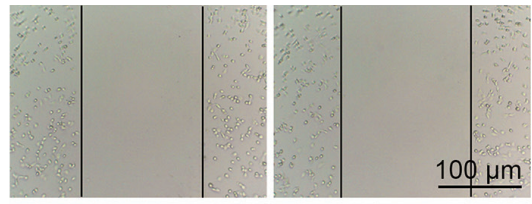

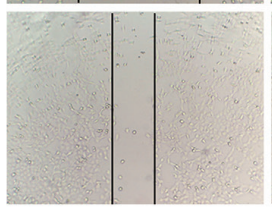

Vector

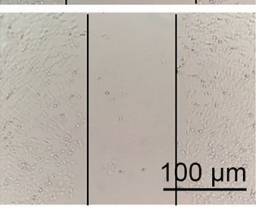

Circ
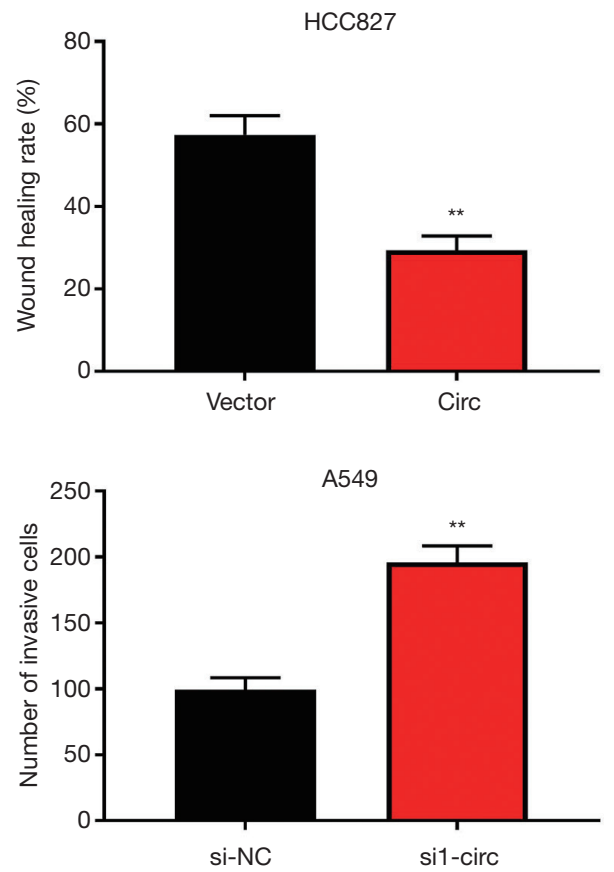

HCC827

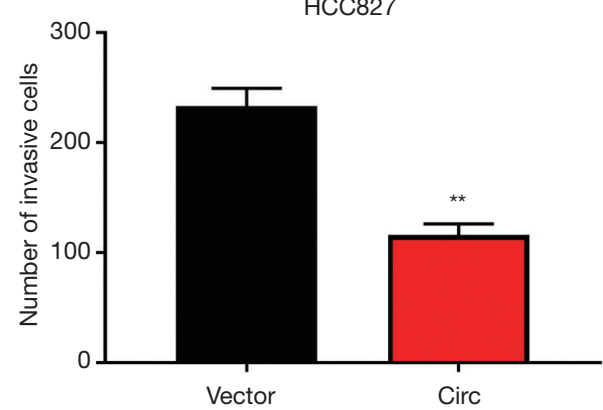

Figure 2 Circ-000881 plays an inhibitory role in A549 and HCC827 cells. (A) Reverse transcription and quantitative polymerase chain reaction (RT-qPCR) was used to measure circ-000881 expression (B) Cell counting Kit-8 assays were performed to assess cell viability. (C) Cell proliferation properties were detected in A549 and HCC827 cells after transfection with si-cric-000881 or si-NC or overexpression plasmid or vector. (D) Wound-healing assays were carried out to investigate the migration capabilities of circ-000881 knockdown A549 cells and circ-000881 overexpression HCC827 cells. (E) Cell invasion ability was examined by conducting Transwell assays. Cells were fixed and stained with $0.1 \%$ crystal violet. ${ }^{* *} \mathrm{P}<0.01$ vs. si-NC or vector group. 
A

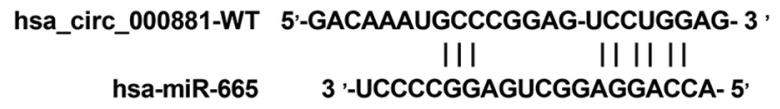

hsa_circ_000881-MUT 5'-GACAAAUGCCCGGAG-AGGACCAG-3 '

B
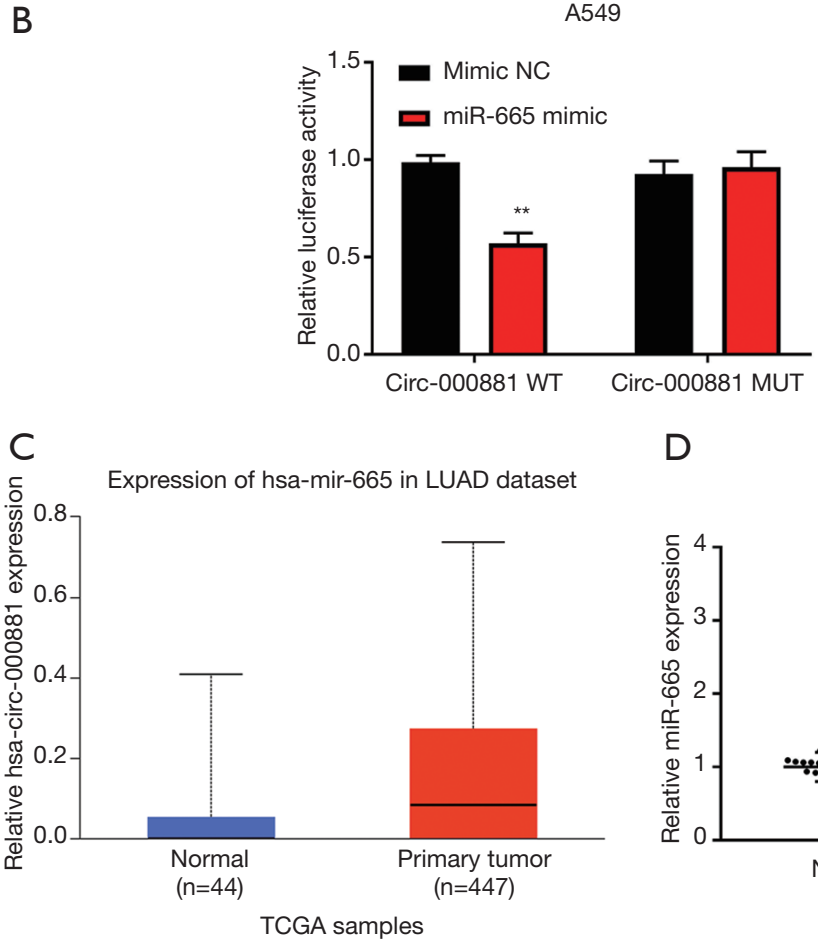

D

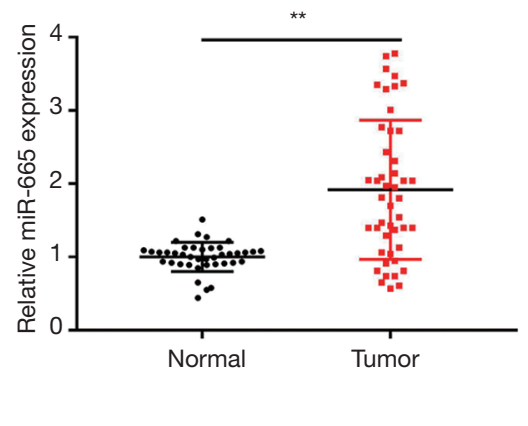

HCC827

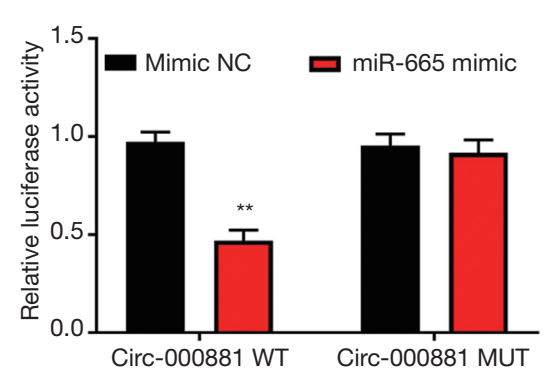

E

Figure 3 Circ-000881 serves as a sponge for miR-665 in lung adenocarcinoma (LUAD). (A) Prediction of possible binding sites between circ-000881 and miR-665 by TargetScan software. (B) The interaction between miR-665 and circ-000881 in A549 and HCC817 cells was analyzed by luciferase reporter assay. (C) Analysis of miR-665 expression in LUAD patients using the The Cancer Genome Atlas (TCGA) database. (D) Expression of miR-665 in 40 pairs of clinical specimens was measured by (RT-qPCR). (E) MiR-665 shows a negative correlation with circ-000881 in LUAD tissues. ${ }^{* *} \mathrm{P}<0.01$ vs. Normal or circ-000881-MUT group.

\section{Circ-000881 exerted inbibitory effects on lung cancer cells by regulating a miR-665/PRICKLE2 axis}

To investigate whether a circ-000881/miR-665/PRICKLE2 regulatory pathway is present in LUAD, we performed a series of assays. The expression of PRICKLE2 in HCC827 cells increased with circ-000881 overexpression, while silencing of circ-000881 decreased PRICKLE2 expression in A549 cells $(\mathrm{P}<0.01$, Figure $5 A)$. We also investigated the effects of circ-000881, miR-665, and PRICKLE2 on the cell proliferation, migration, and invasion abilities of HCC827 cells. We found that overexpression of miR-665 could abolish circ-000881-mediated inhibition of cell malignant biological behavior (Figure 5B-D). Similarly, silencing of PRICKLE2 also abolished circ-000881-mediated inhibition of cell proliferation, migration, and invasion (Figure $5 B-D$ ).
Taken together, these results revealed that circ-000881 suppressed LUAD progression by regulating a miR-665/ PRICKLE2 axis.

\section{Discussion}

With the development of personalized medicine, great advances have been made in the understanding of the carcinogenesis and treatment of NSCLC. However, the clinical outcome for patients is still unsatisfactory due to the lack of specific and efficient early diagnostic approaches (18). LUAD is one of the most common types of NSCLC, especially in women (19). Therefore, mechanistic research of the occurrence and development of LUAD is of great importance for developing new therapeutic strategies for the disease. 


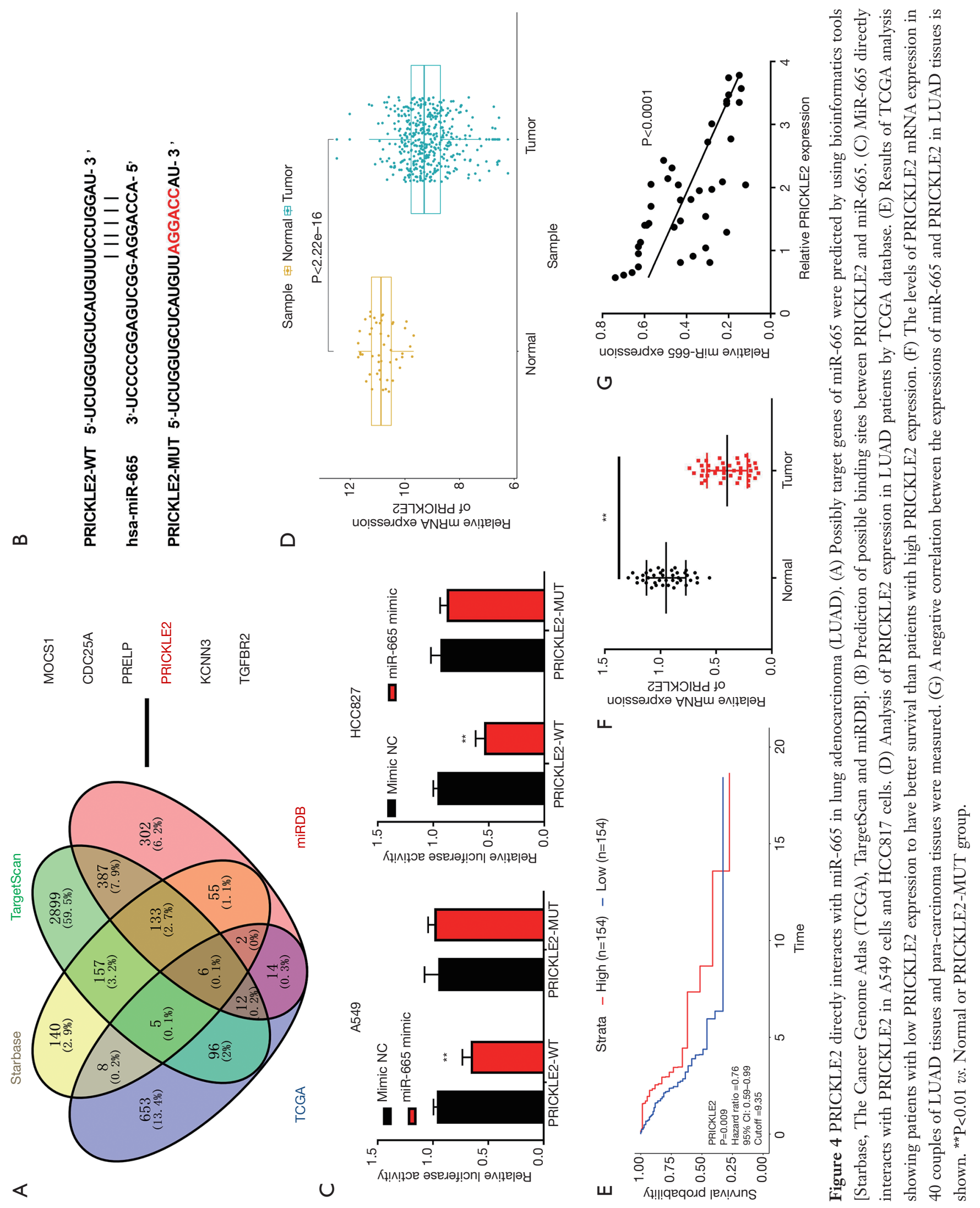


A

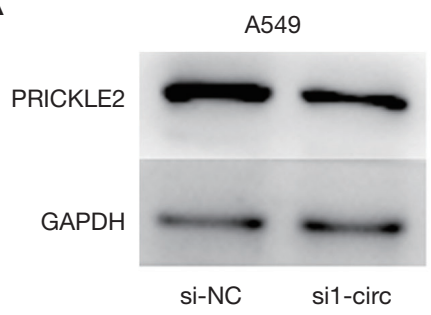

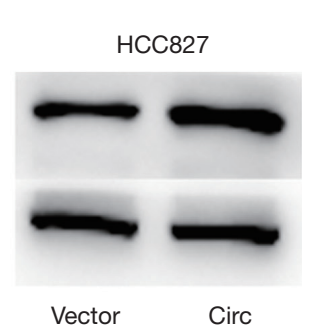

B

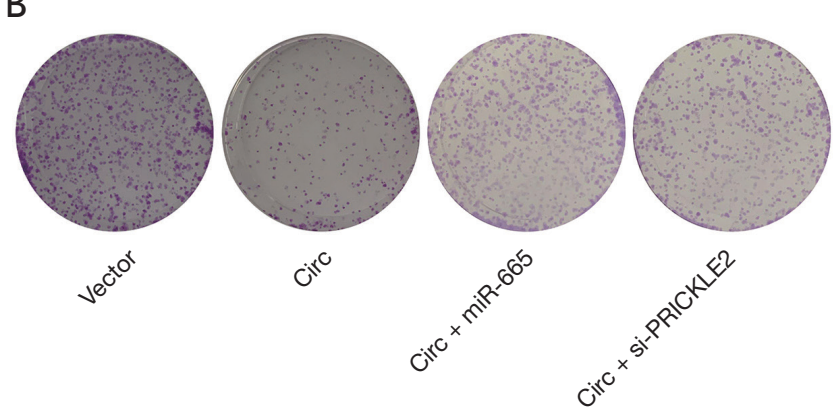

C
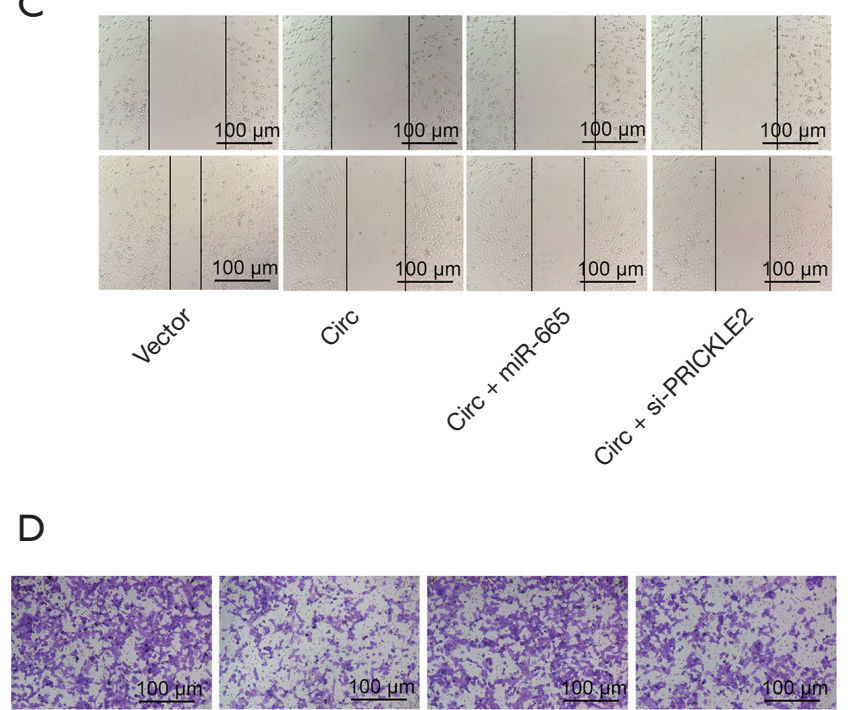

$e^{e^{x}}$

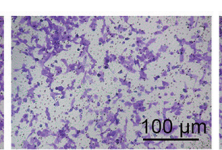

$0^{*}$
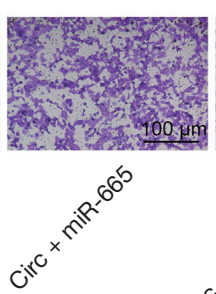
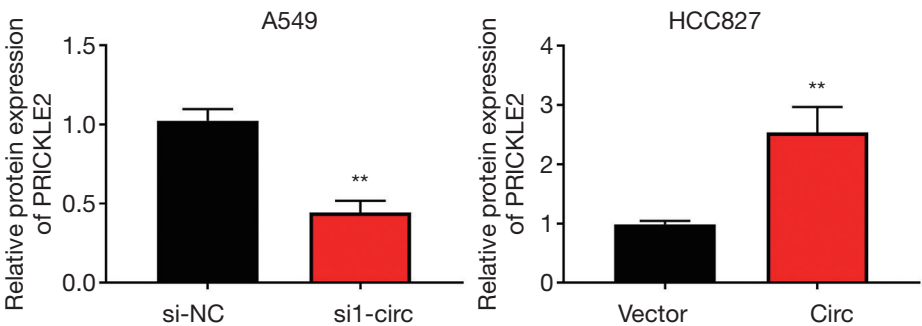
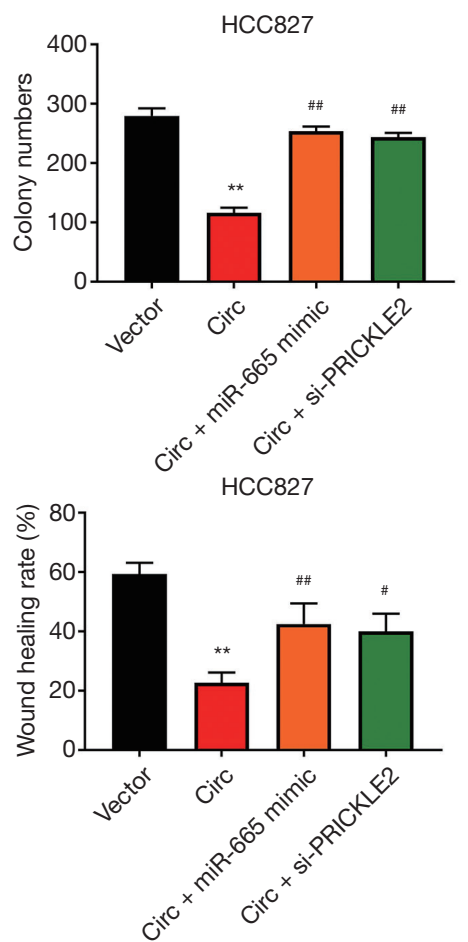

HCC827

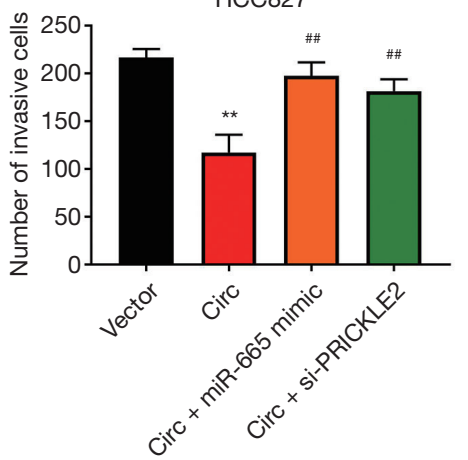

Figure 5 Circ-000881 inhibits the proliferation, migration, and invasion of lung cancer cells via regulating the miR-665/PRICKLE2 axis. (A) Expression of PRICKLE2 at the protein level was detected in HCC827 and A549 cells. (B) Colony formation assay was conducted to examine the proliferation of HCC827 cells. (C) Wound-healing assay was used to detect the migration ability of HCC827 cells. (D) Transwell assay was used to detect the invasion ability of HCC827 cells. Cells were fixed and stained with $0.1 \%$ crystal violet. ${ }^{* *} \mathrm{P}<0.01$ vs. siNC or vector group, ${ }^{\#} \mathrm{P}<0.05$ and $^{\# \#} \mathrm{P}<0.01$ vs. circ group. 
In recent years, circRNAs have been widely researched. The dysregulation of circRNAs has been revealed to lead to the promotion of oncogenesis and progression in several cancers (20-22). We already know that some circRNAs are dysregulated in LUAD, but the understanding of roles of circRNAs in cancer progression is far from sufficient. For this reason, we investigated the influence of circ-000881 on the development of LUAD and attempted to gain an understanding of the pathway and molecular mechanism of its action. Firstly, significant downregulation of circ000881 expression in LUAD tissues and cells was observed. Subsequent functional cellular experiments revealed that overexpression of circ-000881 diminished the migratory, proliferative, and invasive abilities of $\mathrm{HCC} 827$ cells, whereas silencing of circ-000881 accelerated the aggressive malignant biological behavior of A549 cells. These observations confirmed the certain participation of circ0000881 in the progression of LUAD.

Many studies have proved that circRNAs regulate gene expression by sponging miRNAs and play an important part in cancer development (23). For instance, circ-0000515 was recognized to be a tumor promoter in cervical cancer through its sponging of miR-326 (24). Furthermore, Yao et al. identified that circ-LDLRAD3 enhanced the malignant characteristics of pancreatic cancer cells, including their proliferative and migratory abilities, through sponging miR-137-3p (25). During our study of tumor inhibition by circ-000881, miR-665 greatly aroused our interest because of its potential binding site with circ000881, which was revealed by bioinformatics analysis. Many studies have demonstrated miR-665 to be abnormally expressed in tumors such as ovarian cancer (26), gastric adenocarcinoma (27) and hepatocellular carcinoma (28). Liu et al. also found that miR-665 expression was upregulated in small cell lung cancer tissues (29). Xia et al. demonstrated miR-665 up-regulation in NSCLC tissues and cells, with the overexpression of miR-665 promoting cell proliferation, migration, and invasion (30). Likewise, we also found that miR-665 expression was significantly upregulated in LUAD. Furthermore, overexpression of miR-665 abolished the circ-000881-mediated inhibition of lung cancer cell proliferation, migration, and invasion. Taking the above results into consideration, we can reasonably conclude that circ- 000881 suppresses the progression of LUAD by sponging and inhibiting miR-665.

By combining with the 3'-untranslated regions of their downstream target genes, miRNAs can regulate gene expression (2). Among the potential target genes of miR-
665, PRICKLE2 has not been thoroughly studied. The PRICKLE2 gene is located on human chromosome 3 p14 and is expressed in the testes, eyes, and brain. It exerts a variety of functions such as neurite formation, tumor formation, and metastasis (31). Senchenko et al. reported that PRICKLE2 shows methylation/deletion in $22 \%$ of cervical adenocarcinomas and $49 \%$ of squamous cell carcinomas, and has tumor suppression functions, which is promising for the discrimination of these diseases (32). Rudenko et al. found that PRICKLE2 downregulation was associated with the malignant biological behavior of clear-cell renal cell carcinoma (33). Consistent with these reports, we discovered that PRICKLE2 expression was downregulated in LUAD tissues. Also, we found that silencing of PRICKLE2 abolished circ-000881mediated inhibition of cell proliferation, migration, and invasion. Taken together, the findings of this study reveal the involvement of a circ-000881/miR-665/PRICKLE2 network in LUAD.

\section{Conclusions}

Circ-000881 expression is significantly downregulated in LUAD tissues and cells. Circ-000881 plays an inhibitory role in the proliferation, invasion, and migration of LUAD cells via the miR-665/PRICKLE2 pathway. Our findings may be useful in identifying a potential therapeutic target for LUAD.

\section{Acknowledgments}

Funding: This work was supported by the National Natural Science Foundation of China (No. 81672292) and TaiShan Scholar Program of Shandong Province (No. ts201712087).

\section{Footnote}

Reporting Checklist: The authors have completed the MDAR reporting checklist. Available at http://dx.doi.org/10.21037/ atm-21-844

Data Sharing Statement: Available at http://dx.doi. org/10.21037/atm-21-844

Conflicts of Interest: All authors have completed the ICMJE uniform disclosure form (available at http://dx.doi. org/10.21037/atm-21-844). The authors have no conflicts of interest to declare. 
Ethical Statement: The authors are accountable for all aspects of the work in ensuring that questions related to the accuracy or integrity of any part of the work are appropriately investigated and resolved. All procedures performed in this study involving human participants were in accordance with the Declaration of Helsinki (as revised in 2013). Ethics approval for this study was acquired from the Medical Ethics Committee of Qilu hospital, and informed consent was obtained from all participants.

Open Access Statement: This is an Open Access article distributed in accordance with the Creative Commons Attribution-NonCommercial-NoDerivs 4.0 International License (CC BY-NC-ND 4.0), which permits the noncommercial replication and distribution of the article with the strict proviso that no changes or edits are made and the original work is properly cited (including links to both the formal publication through the relevant DOI and the license). See: https://creativecommons.org/licenses/by-nc-nd/4.0/.

\section{References}

1. Bray F, Ferlay J, Soerjomataram I, et al. Global cancer statistics 2018: GLOBOCAN estimates of incidence and mortality worldwide for 36 cancers in 185 countries. CA Cancer J Clin 2018;68:394-424.

2. Sun Z. Circular RNA hsa_circ_0001588 promotes the malignant progression of lung adenocarcinoma by modulating miR-524-3p/NACC1 signaling. Life Sci 2020;259:118157.

3. Jemal A, Siegel R, Xu J, Ward E. Cancer statistics, 2010. CA Cancer J Clin 2010;60:277-300.

4. Feng D, Xu Y, Hu J, et al. A novel circular RNA, hsacirc-0000211, promotes lung adenocarcinoma migration and invasion through sponging of hsa-miR-622 and modulating HIF1- $\alpha$ expression. Biochem Biophys Res Commun 2020;521:395-401.

5. Zhuang H, Wang J, Zhao L, et al. The theoretical foundation and research progress for WBRT combined with erlotinib for the treatment of multiple brain metastases in patients with lung adenocarcinoma. Int $\mathrm{J}$ Cancer 2013;133:2277-83.

6. Kim H, Chung JH. Overview of clinicopathologic features of ALK-rearranged lung adenocarcinoma and current diagnostic testing for ALK rearrangement. Transl Lung Cancer Res 2015;4:149-55.

7. Rybak-Wolf A, Stottmeister C, Glažar P, et al. Circular RNAs in the Mammalian Brain Are Highly Abundant,
Conserved, and Dynamically Expressed. Mol Cell 2015;58:870-85.

8. Memczak S, Jens M, Elefsinioti A, et al. Circular RNAs are a large class of animal RNAs with regulatory potency. Nature 2013;495:333-8.

9. Wang $\mathrm{Y}$, Wang $\mathrm{L}$, Wang $\mathrm{W}$, et al. Overexpression of circular RNA hsa_circ_0001038 promotes cervical cancer cell progression by acting as a ceRNA for miR-337-3p to regulate cyclin- $\mathrm{M} 3$ and metastasis-associated in colon cancer 1 expression. Gene 2020;733:144273.

10. Sang M, Meng L, Sang Y, et al. Circular RNA ciRS-7 accelerates ESCC progression through acting as a miR876-5p sponge to enhance MAGE-A family expression. Cancer Lett 2018;426:37-46.

11. Wang YG, Wang T, Ding M, et al. hsa_circ_0091570 acts as a ceRNA to suppress hepatocellular cancer progression by sponging hsa-miR-1307. Cancer Lett 2019;460:128-138.

12. Xu L, Ma Y, Zhang $\mathrm{H}$, et al. HMGA2 regulates circular RNA ASPH to promote tumor growth in lung adenocarcinoma. Cell Death Dis 2020;11:593.

13. Qu S, Yang X, Li X, et al. Circular RNA: A new star of noncoding RNAs. Cancer Lett 2015;365:141-8.

14. Zhao ZJ, Shen J. Circular RNA participates in the carcinogenesis and the malignant behavior of cancer. RNA Biol 2017;14:514-21.

15. Lu J, Wang YH, Yoon C, et al. Circular RNA circRanGAP1 regulates VEGFA expression by targeting miR$877-3 p$ to facilitate gastric cancer invasion and metastasis. Cancer Lett 2020;471:38-48.

16. Zhong Z, Huang M, Lv M, et al. Circular RNA MYLK as a competing endogenous RNA promotes bladder cancer progression through modulating VEGFA/VEGFR2 signaling pathway. Cancer Lett 2017;403:305-17.

17. Huang WJ, Wang Y, Liu S, et al. Silencing circular RNA hsa_circ_0000977 suppresses pancreatic ductal adenocarcinoma progression by stimulating miR-874-3p and inhibiting PLK1 expression. Cancer Lett 2018;422:7080. Retraction in: Cancer Lett 2018;438:232.

18. Duruisseaux M, Esteller $M$. Lung cancer epigenetics: From knowledge to applications. Semin Cancer Biol 2018;51:116-28.

19. Rodríguez-Martínez Á, Torres-Durán M, Barros-Dios JM, et al. Residential radon and small cell lung cancer. A systematic review. Cancer Lett 2018;426:57-62.

20. Qiu M, Xia W, Chen R, et al. The Circular RNA circPRKCI Promotes Tumor Growth in Lung Adenocarcinoma. Cancer Res 2018;78:2839-51. 21. Wang S, Zhang Y, Cai Q, et al. Circular RNA FOXP1 
promotes tumor progression and Warburg effect in gallbladder cancer by regulating PKLR expression. Mol Cancer 2019;18:145.

22. Huang X, He M, Huang S, et al. Circular RNA circERBB2 promotes gallbladder cancer progression by regulating PA2G4-dependent rDNA transcription. Mol Cancer 2019;18:166.

23. Zhong Y, Du Y, Yang X, et al. Circular RNAs function as ceRNAs to regulate and control human cancer progression. Mol Cancer 2018;17:79.

24. Tang Q, Chen Z, Zhao L, et al. Circular RNA hsa_ circ_0000515 acts as a miR-326 sponge to promote cervical cancer progression through up-regulation of ELK1. Aging 2019;11:9982-99.

25. Yao J, Zhang C, Chen Y, et al. Downregulation of circular RNA circ-LDLRAD3 suppresses pancreatic cancer progression through miR-137-3p/PTN axis. Life Sci 2019;239:116871.

26. Zhou P, Xiong T, Yao L, et al. MicroRNA-665 promotes the proliferation of ovarian cancer cells by targeting SRCIN1. Exp Ther Med 2020;19:1112-20.

27. Tang H, Long Q, Zhuang K, et al. miR-665 promotes the progression of gastric adenocarcinoma via elevating FAK activation through targeting SOCS3 and is negatively regulated by lncRNA MEG3. J Cell Physiol 2020;235:4709-19.

Cite this article as: Huang C, Yue W, Li L, Li S, Gao C, Si L, Qi L, Cheng C, Lu M, Chen G, Cui J, Zhao R, Li Y, Tian H. Circular RNA hsa-circ-000881 suppresses the progression of lung adenocarcinoma in vitro via a miR-665/PRICKLE2 axis. Ann Transl Med 2021;9(6):498. doi: 10.21037/atm-21-844
28. Mohamed AA, Omar AAA, El-Awady RR, et al. MiR-155 and MiR-665 Role as Potential Non-invasive Biomarkers for Hepatocellular Carcinoma in Egyptian Patients with Chronic Hepatitis C Virus Infection. J Transl Int Med 2020;8:32-40.

29. Liu R, Zhang L, Xu Z, et al. MiR-665 Promotes the Biological Behavior of Small Cell Lung Cancer by Targeting LLGL1. Zhongguo Fei Ai Za Zhi 2020;23:223-32.

30. Xia J, Li D, Zhu X, et al. Upregulated miR-665 expression independently predicts poor prognosis of lung cancer and facilitates tumor cell proliferation, migration and invasion. Oncol Lett 2020;19:3578-86.

31. Katoh M. Identification and characterization of human PRICKLE1 and PRICKLE2 genes as well as mouse Prickle1 and Prickle2 genes homologous to Drosophila tissue polarity gene prickle. Int J Mol Med 2003;11:249-56.

32. Senchenko VN, Kisseljova NP, Ivanova TA, et al. Novel tumor suppressor candidates on chromosome 3 revealed by NotI-microarrays in cervical cancer. Epigenetics 2013;8:409-20.

33. Rudenko E, Gerashchenko G, Lapska Y, et al. PPM1M and PRICKLE2 are potential tumor suppressor genes in human clear-cell renal cell carcinoma. Biopolymers and Cell 2014;30:229-33.

(English Language Editor: J. Reynolds) 